\title{
PROMESA, VARIEDAD DE POLINIZACIÓN LIBRE DE CÁRTAMO PARA LA REGIÓN DE LAS HUASTECAS
}

\author{
PROMESA, OPEN POLLINATED SAFFLOWER VARIETY FOR THE HUASTECAS REGION
}

\author{
Juan Valadez-Gutiérrez ${ }^{1}$ y José E. Cervantes-Martínez²
}

\begin{abstract}
'Campo Experimental Las Huastecas, Instituto Nacional de Investigaciones Forestales Agrícolas y Pecuarias (INIFAP). Km 55 Carretera TampicoMante, 89610. Villa Cuauhtémoc, Municipio de Altamira, Tamaulipas. Tel. 015538718700 Ext. 83302. ²Facultad de Agronomía, Unidad Académica Multidisciplinaria Mante. Universidad Autónoma de Tamaulipas. Av. Juárez, 89840. Jardín, Cd. Mante, Tamaulipas.
\end{abstract}

*Autor para correspondencia (valadez.juan@inifap.gob.mx)

En la región de Las Huastecas, México, el cártamo (Carthamus tinctorius L.) se integra a una rotación de cultivos, que permite efectuar dos cosechas en un periodo de 10 meses, lo cual incrementa los ingresos del productor y hace más eficiente el uso del suelo y del ambiente. Sin embargo, existen pocas variedades de alto potencial de rendimiento de grano, con aceptable contenido de aceite y resistentes a las principales enfermedades, atributos que caracterizan a la variedad Promesa.

El proceso de mejoramiento genético se inició en el ciclo 0-I 2000-2001, con la siembra de un vivero constituido por 30 líneas genéticas, introducidas de EE.UU. En forma preliminar, la línea PI-537693 se seleccionó con base en su aspecto de planta, potencial productivo y resistencia a enfermedades. En evaluaciones de rendimiento efectuadas durante el periodo 2002 a 2004, ese genotipo mostró superioridad con respecto a las dos mejores líneas (P-250199 y W-9048) disponibles en esa época. Esta superioridad se confirmó en experimentos establecidos en los ciclos de O-I (2008-2009 a 2011-2012), periodo durante el cual las evaluaciones se alternaron con ciclos de selección masal. El tercer ciclo de selección masal [PI-537693(P3)] estabilizó su producción de grano en áreas de temporal, 1425 $\mathrm{kg} \mathrm{ha}^{-1}$ en promedio y $2373 \mathrm{~kg} \mathrm{ha}^{-1}$ con riego o humedad residual (Cuadro 1), por lo que se decidió registrarla como una nueva variedad de polinización libre (Valadez-Gutiérrez y Montoya-Coronado, 2010). Este genotipo se registró ante el Servicio Nacional de Inspección y Certificación de Semillas (SNICS), con el número CAR-010-181115 bajo la denominación Promesa (SNICS, 2016).

El contenido de aceite de Promesa es de $34.0 \%$, con predominio del ácido linoleico en proporción 3:1 con respecto al ácido oleico (Aceites Especiales TH, Com. Pers.)' ${ }^{1}$. Esta variedad posee excelente adaptación a las condiciones de producción de la región de Las Huastecas, donde presenta resistencia a las principales enfermedades foliares y del capítulo. Al comparar su rendimiento de grano con el de las variedades más recientes, superó a Guayalejo en $12 \%$, a RC-1005-L en $23 \%$, a RC-1033-L en $13 \%$ y en $26 \%$ a CIANO Oleica (Cuadro 1).

En cuanto a la susceptibilidad a enfermedades foliares (Alternaria carthami Chowd. y Puccinia carthami Corda.), Promesa presentó baja incidencia (2.7), similares a Guayalejo y RC-1033-L $(2,6)$ pero menores a las variedades RC-1033-L y

${ }^{1}$ Aceites Especiales TH. Abril 2015. Av. Eje Norte Sur 451 Cd. Industrial Morelia, Mich. México. C.P. 58200 Tel +52 (443)204 1032 www. aceites-cspecialesth.mx

Cuadro 1. Rendimiento $\left(\mathrm{kg} \mathrm{ha}^{-1}\right)$ de Promesa y cuatro genotipos adicionales de cártamo en seis ambientes de validación.

\begin{tabular}{|c|c|c|c|c|c|c|c|c|}
\hline \multirow{2}{*}{ Genotipo } & 2008-09 & $2009-10$ & $2009-10$ & $2010-11$ & $2010-11$ & $2011-12$ & \multirow{2}{*}{$\begin{array}{l}\text { Prom. } \\
\text { (kg/ha) }\end{array}$} & \multirow{2}{*}{$\begin{array}{c}\text { Dif. vs. } \\
\text { Promesa (\%) }\end{array}$} \\
\hline & CEHUAS $^{+}$ & CEHUAS & Col. Campo Nvo. ${ }^{++}$ & CEHUAS & Tantoyu-quitat+ $^{++}$ & CEHUAS & & \\
\hline Promesa & $1888 a$ & $1436 a$ & $885 a$ & $2373 a$ & $1178 a$ & $793 a$ & 1425 & 0 \\
\hline Guayalejo & 1920 a & $1020 \mathrm{~b}$ & $775 b$ & 2081 a & 1178 a & $586 b$ & 1260 & -12 \\
\hline RC-1005-L & $1789 b$ & $1089 b$ & $657 b$ & 1196 c & $1196 a$ & $667 b$ & 1099 & -23 \\
\hline RC-1033-L & $1700 \mathrm{~b}$ & 1536 a & $1124 \mathrm{a}$ & $1556 b$ & $1020 \mathrm{~b}$ & $488 \mathrm{c}$ & 1237 & -13 \\
\hline CIANO Oleica & $1835 a$ & 912 c & $588 \mathrm{c}$ & $1578 b$ & $1090 \mathrm{~b}$ & $279 d$ & 1047 & -26 \\
\hline
\end{tabular}

${ }^{+}$CEHUAS: Campo Experimental Las Huastecas; ${ }^{++}$Localidades del Municipio de González, Tamps. Valores con letras iguales en las columnas, no son estadísticamente diferentes (Tukey, 0.05). 
CIANO Oleica (3.4 y 4.6 respectivamente).

En siembras efectuadas durante la primera quincena del mes de diciembre (periodo óptimo de siembra) la floración de Promesa inicia a los 97 días después de la siembra (dds), y la madurez fisiológica del grano ocurre a los 135 dds (Cuadro 2), por lo que se considera de ciclo intermedio. Posee un hábito de crecimiento determinado, con una altura promedio de $100 \mathrm{~cm}$. Los limbos de las hojas son de color verde oscuro de forma alargada con borde aserrado, cuya base es angosta, que se ensancha en sus partes media y superior. En densidades de población entre 100 a 125 mil plantas/ha, las plantas presentan una ramificación abundante, que se concentra en los dos tercios superiores. El tallo es erecto, sólido y resistente al acame.

El capítulo, en su madurez de cosecha, tiene una apariencia semi-redondeada; los granos están protegidos por abundante pubescencia color blanco lustroso. En promedio produce 25 capítulos por planta y 40 granos por capítulo. El diámetro promedio de los capítulos es $2.5 \mathrm{~cm}$. Las flores frescas son de color amarillo y a la cosecha, el 53 $\%$ de las flores permanecen con dicho color y el resto se tornan rojizas (Figura 1). El polen es de color amarillo. Las brácteas se ubican en la parte externa de los capítulos envolviéndolos por completo. La semilla es un aquenio de forma oval angulosa de $7.3 \mathrm{~mm}$ de longitud y $4.0 \mathrm{~mm}$ de ancho en su parte media. La semilla normal contiene $35 \%$ de pericarpio, $65 \%$ de almendra, sin vilano. El peso promedio de 1000 granos es de $43 \mathrm{~g}$, con un peso específico de $574 \mathrm{~g} \mathrm{~L}^{-1}$ de semilla a $7 \%$ de humedad.

El área de adaptación para esta nueva variedad de cártamo comprende la región de Las Huastecas y otras con condiciones agroclimáticas similares: clima cálido subhúmedo con lluvias en verano (AW") y semiseco muy cálido

Cuadro 2. Características agronómicas de Promesa y variedades comerciales de cártamo.

\begin{tabular}{lccccc}
\hline \multirow{2}{*}{ Variedad } & \multicolumn{2}{c}{ Días a } & Reacción a Roya ${ }^{+}$ & $\begin{array}{c}\text { Altura de planta } \\
(\mathrm{cm})\end{array}$ & $\begin{array}{c}\text { Peso específico } \\
\left(\mathrm{g} \mathrm{L}^{-1}\right)\end{array}$ \\
\cline { 2 - 3 } Promesa & Floración & Madurez fisiológica & Rearn & 118 & 574 \\
Guayalejo & 97 & 135 & 2 & 120 & 566 \\
Tantoán 91 & 100 & 140 & 5 & 115 & 523 \\
Mante 81 & 96 & 138 & 2 & 135 & 550 \\
\hline
\end{tabular}

†Escala 0 a 10, 0: resistente; 10: muy susceptible.

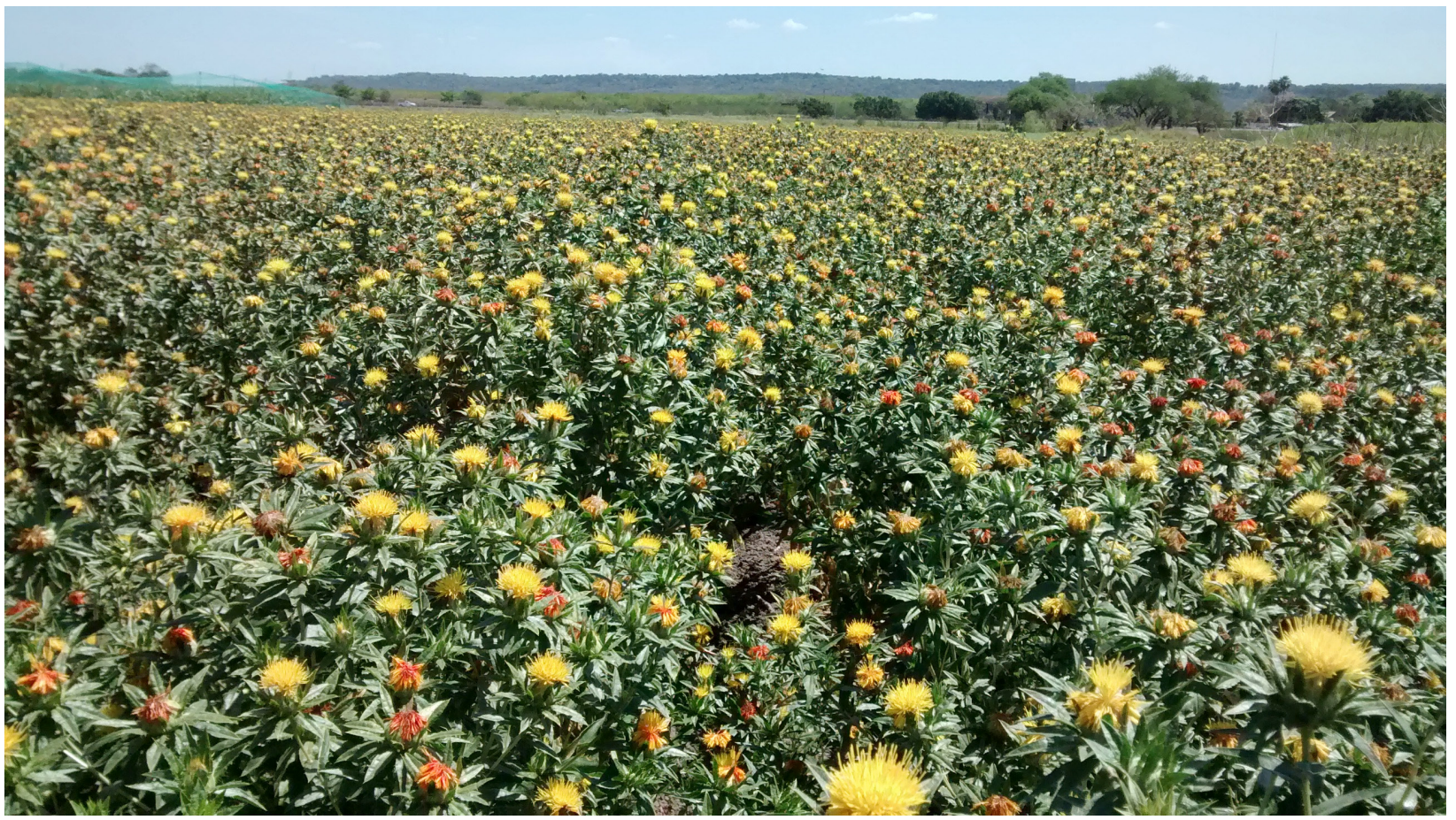

Figura 1. Detalle de floración de la variedad de cártamo Promesa, en condiciones de labranza de conservación. 
y cálido (BS1) en condiciones de riego (INEGI, 1994). Para obtener buenos rendimientos es necesario aplicar las especificaciones descritas por Valadez (2015). En el Campo Experimental Las Huastecas, se dispone de semilla original para producir las categorías básica y registrada que requieran los productores o compañías de semillas interesadas.

\section{BIBLIOGRAFÍA}

INEGI, Instituto Nacional de Estadística y Geografía (1994) Anuario Estadístico del Estado de Tamaulipas. Instituto Nacional de Estadística, Geografía e Informática, Aguascalientes, Ags. México. pp:7-8,11.

SNICS, Servicio Nacional de Inspección y Certificación de Semillas (2016) Ca- tálogo Nacional de Variedades Vegetales. No. 10. Servicio Nacional de Inspección y Certificación de Semillas. Secretaría Agricultura Ganadería Desarrollo Rural Pesca y Alimentación. Cd. de México. 29 p 5. http://snics.sagarpa.gob.mx/Documents/2016/ CNVV\%202016\%20completo.pdf (Septiembre, 2016).

Valadez G. J. (2015) Guía para cultivar cártamo optimizando captación de agua para siembras de temporal en Las Huastecas. Folleto Técnico No. MX-0-310301-52-03-14-09-40. Centro de Investigación Regional del Noreste. Campo Experimental Las Huastecas. Instituto Nacional de Investigaciones Forestales, Agrícolas y Pecuarias. Villa Cuauhtémoc, Tamaulipas. $25 \mathrm{p}$.

Valadez-Gutiérrez J. y L. Montoya-Coronado (2010) Rendimiento y características agronómicas de siete nuevos genotipos de cártamo con y sin control de plagas y enfermedades en el sur de Tamaulipas. In: XXXV Congreso Nacional de la Ciencia del Suelo y XIII Congreso Internacional de Ciencias Agrícolas, Simposio en Agricultura Sustentable. 25-29 oct. 2010. Instituto de Ciencias Agrícolas, Universidad Autónoma de Baja California. Mexicali, Baja California. pp:1151-1156. 